Gut, 1976, 17, 624-632

\title{
Adenocarcinoma of the stomach: radiological and pathological correlation of effects of treatment with fast neutrons
}

\author{
D. KINGSLEY' A. GAD ${ }^{2}$, AND MARY CATTERALL
}

From the Departments of Radiodiagnosis and Histopathology of the Royal Postgraduate Medical School and the Medical Research Council's Cyclotron Unit, Hammersmith Hospital, London

SUMMARY During the past six years 40 patients have been treated with fast neutrons for inoperable adenocarcinoma of the stomach. A number of these patients have been studied in detail both radiologically and pathologically with regard to the effects of fast neutrons on both the tumour and normal stomach. After treatment there was a constant reduction in size of the stomach capacity when compared with a control group and the motility of the stomach was lost. Mucosal changes, which were noted radiologically, appeared to be dose related. Histologically most of the tumour was destroyed. Small clumps of cells were, however, found in all except one of the patients but only in areas of the stomach which had not received the standard dose. Marked fibrosis occurred and it is our impression that the amount of fibrosis is in excess of that seen with other forms of radiotherapy.

The management of adenocarcinoma of the stomach remains as much of a problem today as it has ever been. In the United Kingdom it is the third commonest tumour (Burn, 1971), while in Finland, Iceland, and Japan the death rate from this disease is the highest in the world (Inberg et al., 1972).

The treatment of choice is resection where possible. However, the diagnosis is frequently made too late and at laparotomy the disease is found to be inoperable or unresectable in as many as $40-60 \%$ of cases (Lumpkin et al., 1964; ReMine and Priestley, 1966; Zacho and Fischermann, 1966; Gilbertson, 1969; Burn, 1971). In these cases management is then limited to symptomatic treatment.

Of the methods available, radiation with photons (X or gamma rays) appears to be of limited value because, although any tumour can be destroyed by ionising radiation if a sufficiently high dose is given, radiation is not selective in its action and affects normal as well as tumour tissue. Adenocarcinoma of the stomach responds relatively poorly and therefore a high dose is required. In treating such patients

'Present address: Lysholm Radiological Department, The National Hospital for Nervous Diseases, Queen Square, London, W.C.1.

${ }^{2}$ Present address: Department of Histopathology and Cytology, Falun Hospital, S-791 01, Falun, Sweden.

Accepted for publication 19 May 1976 it is inevitable that the normal tissue through which the beam passes will be subject to such radiation.

During the past six years, approximately 40 patients have been referred to the Medical Research Council's cyclotron unit at Hammersmith Hospital with inoperable adenocarcinoma of the stomach. These patients were treated with a course of $7.5 \mathrm{MeV}$ fast neutrons to the tumour and its lymph node bed, the average size of the treated area being $15 \times 10 \times$ $10 \mathrm{~cm}$. The results of this treatment (Catterall et al., 1975), show that considerable symptomatic relief can be given without much inconvenience to the patient, at least in the short term. Longer periods of follow-up show that radiation caused the macroscopic disappearance of the tumour but adversely affected the normal stomach.

The purpose of this paper is to examine further the remarkable shrinkage which occurs after neutrons and to correlate the pathological appearances with the histology and the dose received at various sites throughout the stomach.

\section{Methods}

Assessment of the patient's progress after radiation was based on a series of barium meals undertaken at intervals before and after treatment started.

The initial barium meal was used to plan the fields of treatment. In some patients who had undergone 
laparotomy, surgical clips were placed around the mass to outline the macroscopic extent of the tumour more accurately.

Follow-up films were used to assess gastric motility, overall changes in size of the tumour and of the stomach capacity, and to note any consistent pattern of change in outline which might be correlated with histopathology at subsequent surgery or necropsy.

One film from each follow-up examination was selected for an assessment of overall stomach size and the effect of irradiation on the tumour mass. As the patients were treated in the sitting position, an erect anteroposterior view was used where possible. Occasionally, this view was found to be unsatisfactory for technical reasons and a supine view had to be used.

The outline of the stomach from oesophagus to duodenal cap was traced onto sectional tracing paper. The position of the stomach relative to the bony structures was established by tracing the outline of the vertebral bodies and superimposing each subsequent $x$-ray relative to the vertebral column (Fig. 1).

The stomach capacity was assessed by counting all large squares and fractions of squares greater than $50 \%$ within the stomach outline from cardia to

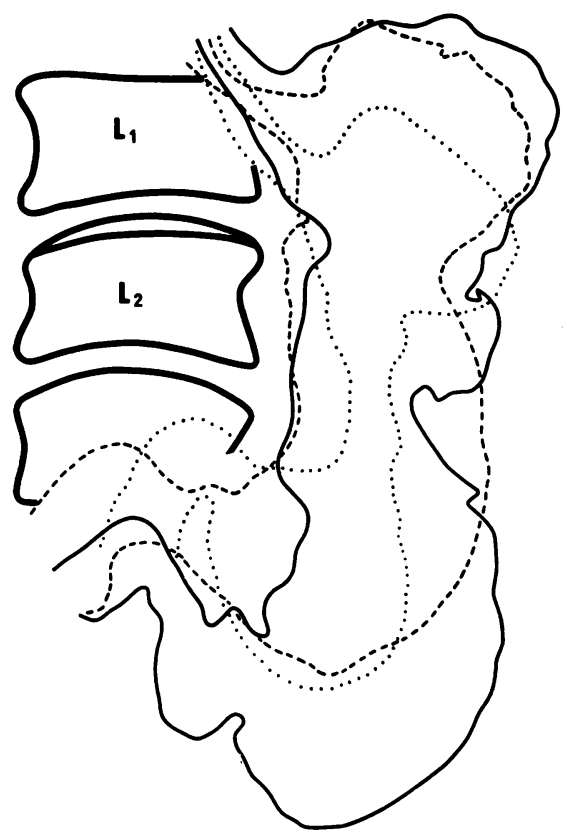

Fig. 1 Outline of stomach obtained from comparable serial radiographs showing change in shape after treatment with fast neutrons. $-=$ during treatment, $\ldots=$ six months after treatment started, . . . . = 10 months after treatment started. pylorus. The stomach capacity at the first examination was taken as $100 \%$. A correction factor was applied to each subsequent film using the width of the vertebral bodies to assess magnification. The final stomach size was calculated as a percentage of the first film of the series. The control group consisted of five patients with other non-gastric conditions who had had at least three barium meals.

Assessment of any consistent radiological changes noted during life was correlated with the radiological appearances and histological findings after death and also with the dose recieved by different parts of the stomach in different patients.

Since the stomach lies obliquely across the abdomen, the fundus, body and pylorus are not in the same vertical or horizontal plane. Consequently, in any given patient the amount of radiation varied from one part of the stomach to another. The actual dose delivered to each area of the stomach was calculated using a combination of planning charts, simulator films, body section diagrams (Takahashi, 1969), and radiation changes to the vertebral bodies.

At necropsy, the stomach, liver, spleen, and kidneys and the relevant vertebral bodies were removed in one block. Surgical clips were placed at intervals of approximately $5 \mathrm{~cm}$ around the greater and lesser curve. The stomach contents were evacuated and replaced by dilute barium solution and radiographs of the whole block were taken (Fig. 2). Blocks were taken from areas of oesophagus, stomach, and proximal part of the duodenum and corresponded to the surgical clips placed at necropsy (Fig. 3). These were fixed in $10 \%$ neutral buffered

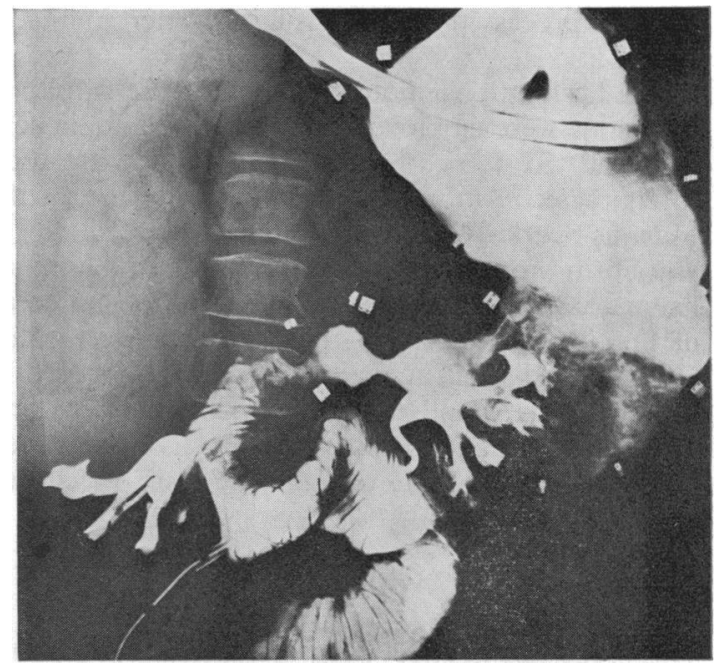

Fig. 2 Radiograph of block of tissue removed at necropsy. The gastrointestinal tract and kidneys have been filled with barium. 


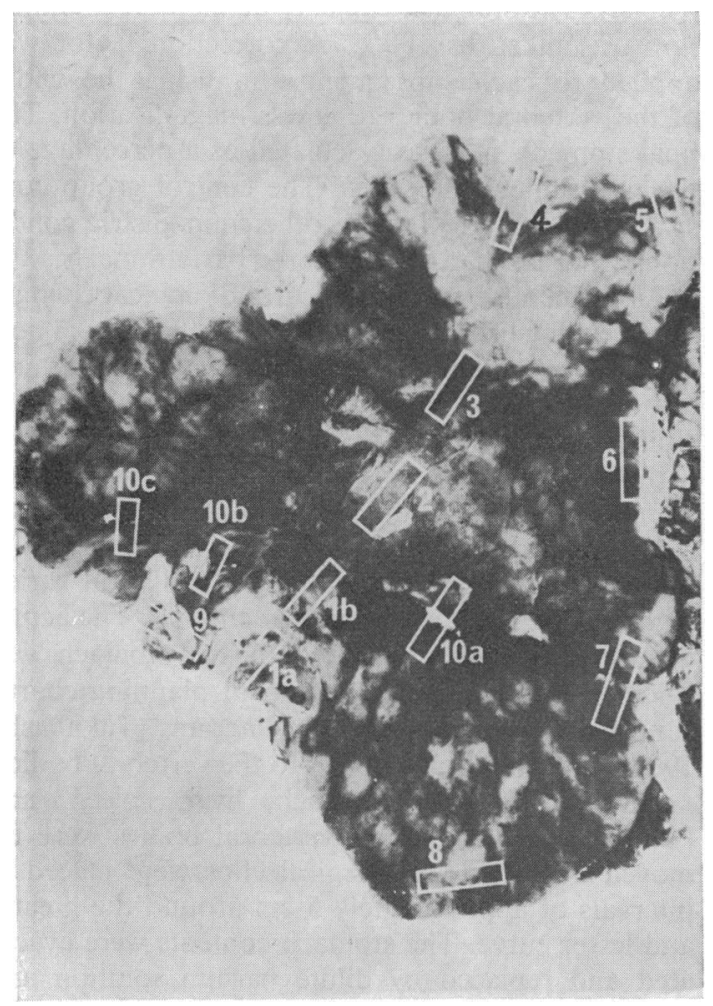

Fig. 3 Stomach opened along the greater curvature showing typical distribution of the blocks taken for histological examination. Oesophagus is at $1 a$ and duodenum at 4.

foralin, processed in the usual manner and sectioned at $5 \mu$.

In addition to haemotoxylin and eosin, the following stains were employed in all cases: Periodic acid Schiff (PAS), Grocott, and Gram stains were used to characterize mixed bacterial and fungal growth; Martius-Scarlet-Blue (MSB) demonstrated fibrin and fibrin thrombi in blood vessel walls and lumina; haematoxylin Van Giesen (HVG) mapped out areas of fibrosis in the wall of the stomach; Alcian blue periodic acid Schiff sequence (AB/PAS) was used in assessing the extent of invasion of mucus secreting adenocarcinoma, especially in the dissociated cell or linitis plastica type, through the wall of various viscera studied.

\section{Results}

This study has in effect been divided into two parts. The first has been concerned with the effects of radiation on stomach capacity using serial radiographs. The second has been a more detailed study of these and other radiological changes with relation to the histopathological appearances at necropsy.

\section{Radiological findings}

\section{STOMACH CAPACITY}

Only 10 patients have been found suitable for assessment of stomach capacity. Of the 10 patients, necropsy was performed on four and total gastrectomy on one. All had at least three barium meals, one of which was undertaken at the time of treatment. In all these cases the whole of the stomach had been in the area of irradiation.

In the patients with neutron treated carcinoma there was a constant reduction in the capacity of the stomach (Fig. 4). This change appeared almost im-

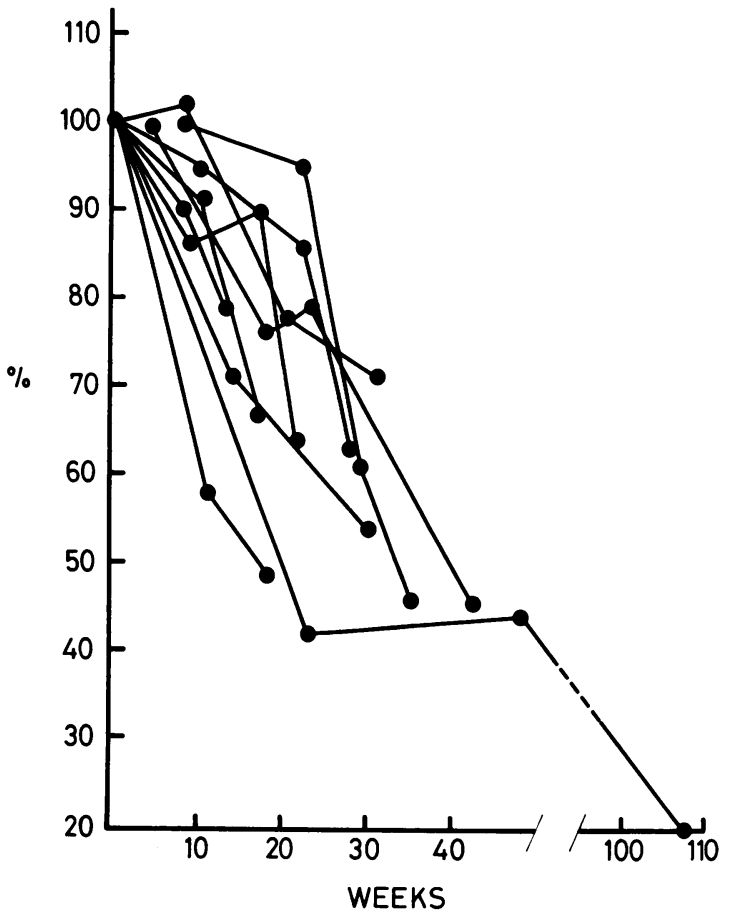

Fig. 4 The ordinate represents the area of the stomach on serial barium studies expressed as a percentage of the examinations before or early in treatment in patients with neutron treated adenocarcinoma.

mediately after treatment and progressed over the subsequent months. Four out of the 10 showed a reduction of at least $50 \%$ in the stomach capacity, while in one patient only $20 \%$ of the original stomach area remained two years after treatment. By comparison, the control group showed a very different picture over a much longer period, the 
stomach sizes varying by less than $10 \%$ in any one person (Fig. 5).

\section{MUCOSAL APPEARANCES}

The mucosal changes were assessed in 10 patients all of whom came to necropsy. After treatment there were two main changes in pattern. In the first there was a loss of the normal parallel rugal pattern (Fig. 6), which was replaced by a flat surface with rather shallow clefts spaced fairly widely apart. This change was seen between 10 and 20 weeks after treatment in areas of the stomach which received the full dosethat is, $1560 r$ in 12 fractions over 26 days. In areas which received less radiation this change appeared later and lasted longer.

The second type of mucosal pattern was a heapedup appearance with irregularly placed clefts (Fig. 7). This change was seen only in areas which had received a full or larger dose than the standard one and appeared after the previously noted change.

Motility of the stomach was reduced in all patients and was notably absent from 30 weeks onwards after treatment. Despite irradiation of the cardia, oesophageal reflux was seen in only one patient whose tumour involved the gastro-oesophageal junction.

${ }^{3}$ This was previously stated as $1440 r$ (Bewley, 1976).

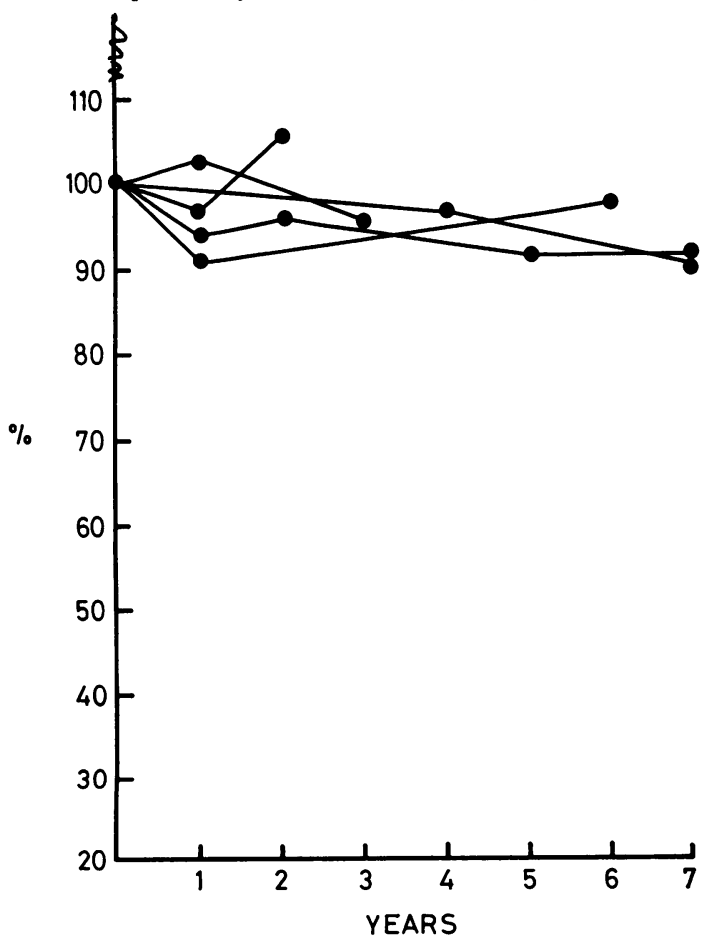

Fig. 5 Stomach size of the control group of patients. The ordinate represents the size of the stomach as a percentage of its area at the first examination.
HISTOLOGICAL FINDINGS

There was macroscopical evidence of tumour in only one patient but histological evidence of tumour cells in all except one. Most specimens showed thickening of the stomach wall only in the area previously known to contain tumour. Four of these had had laparotomy before irradiation at which an inoperable mass had been noted.

Most of the tumour cells appear to have been viable, although much distorted, and tumour necrosis was evident in some areas (Fig. 8). However, in stomachs with extensive widespread infiltration by tumour cells many of these showed mitotic activity with bizarre forms. In one patient who had a second course of neutrons and so probably had an exceptionally high dose, tumour was found in the sub-mucosa outside the second field of irradiation, while the original site of the tumour showed no evidence of carcinoma.

Radiation effects in the stomach wall were classified as 'early' when blood vessel dilatation, fibrin thrombi, and radiation fibroblasts were seen (Figs. 9, $10,11)$ and 'late' when mature fibrous tissue was present and the blood vessel showed intimal thickening and luminal obliteration (Fig. 12).

The early changes were most marked in those patients who died within three to four months of beginning treatment and were particularly prominent in the patients who received a high dose. Late changes were seen in the seven patients who survived longer than five months and were most marked in areas which received a high dose. However, neither early nor late changes were mutually exclusive.

Mixed infection with bacteria and fungi was present in seven of 10 patients (Fig. 13). Its presence was not related either to the radiation dose or to the length of survival.

In a number of cases there was considerable autolysis of the mucosa; this was probably due to the length of time between necropsy and death. Ulceration was present both early and late and sometimes was accompanied by mixed infection. It was particularly prominent in areas of the stomach which had received high doses of irradiation.

\section{Discussion}

Most reports of irradiation of the stomach have concerned the use of $x$ or Gamma-rays, and there is no reason to think that fast neutrons would produce changes any different from those already described. Indeed, Hirose in 1967 compared the effects of $14 \cdot 1$ $\mathrm{MeV}$ neutrons and $x$-rays in experimental radiation damage to the digestive tract in mice and came to the conclusion that, although fast neutrons had a greater inhibitory effect on mitotic activity, there was no 

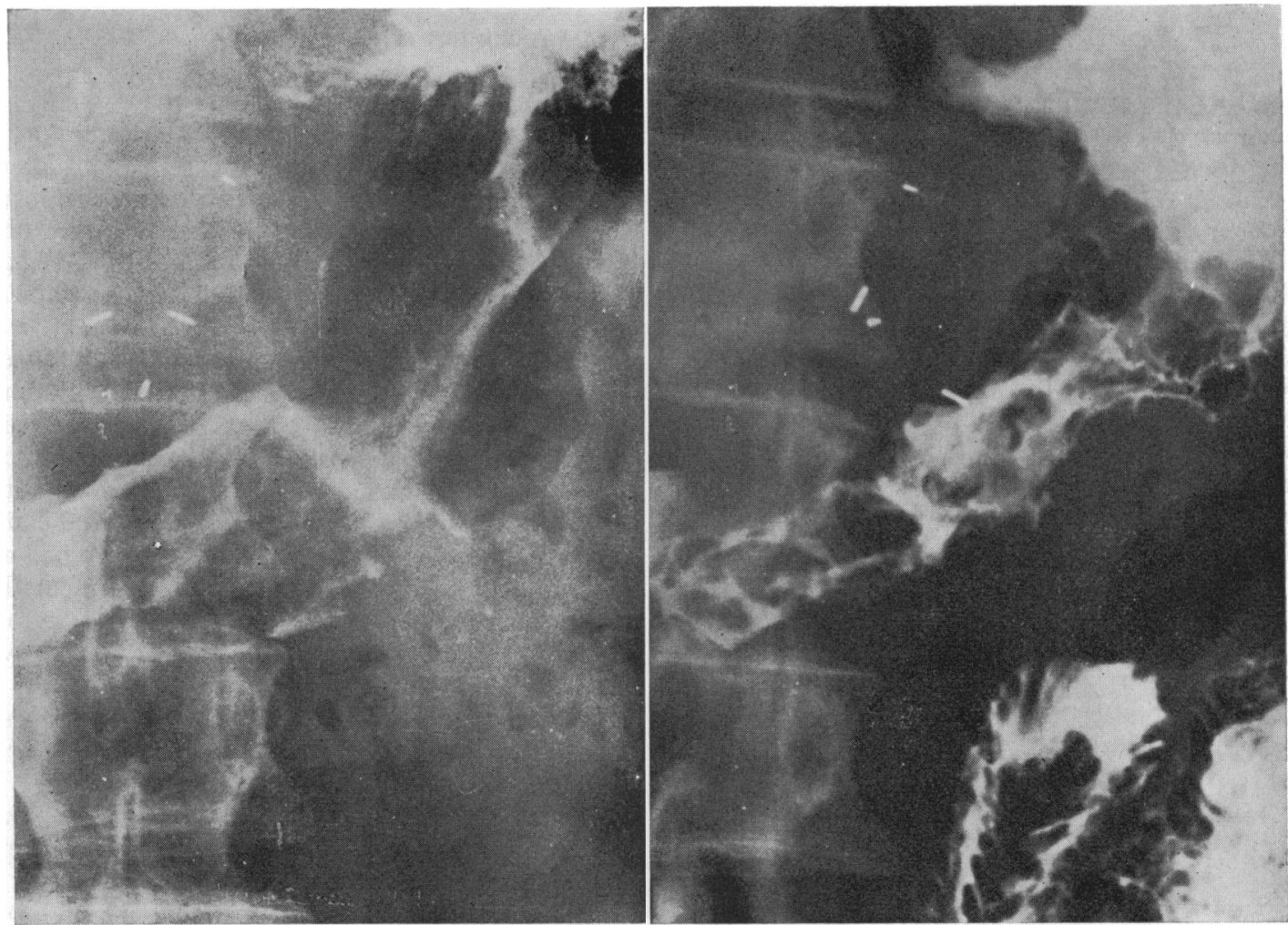

Fig. 6

Fig. 7

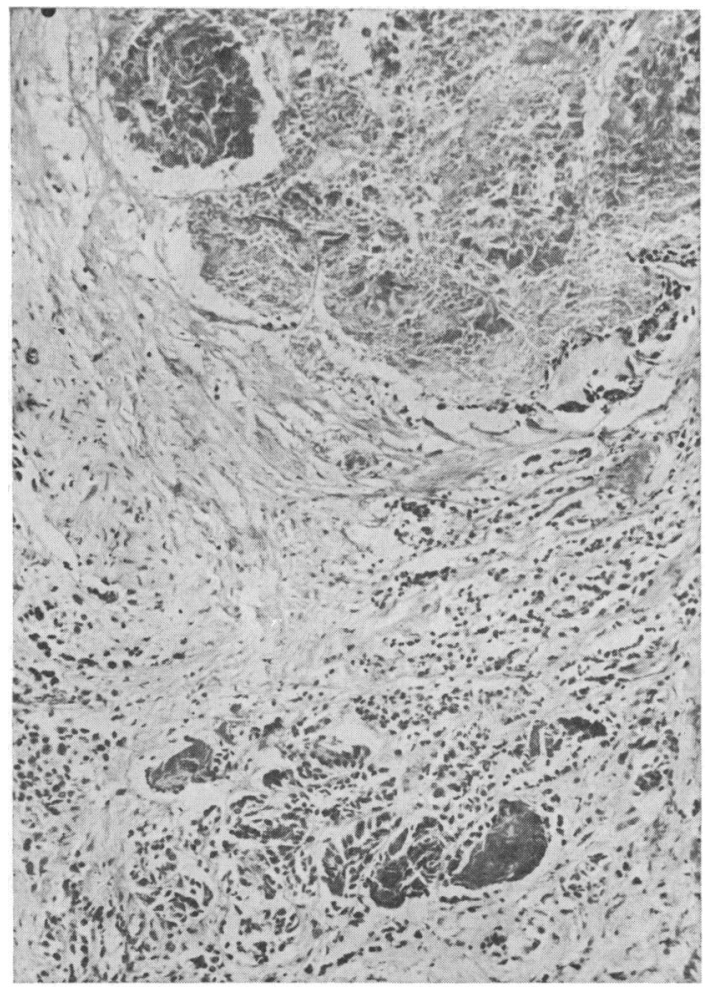

Fig. 6 Appearances of the stomach between 10 and 20 weeks after treatment started.

Fig. 7 Distorted mucosal appearance seen only after treatment with a high dose.

Fig. 8 Extensive tumour necrosis most marked in the upper right corner. Residual viable tumour cells together with round cell infiltrate are seen in the surrounding mature fibrous tissue. $H$ and $E, \times 90$.

Fig. 8 


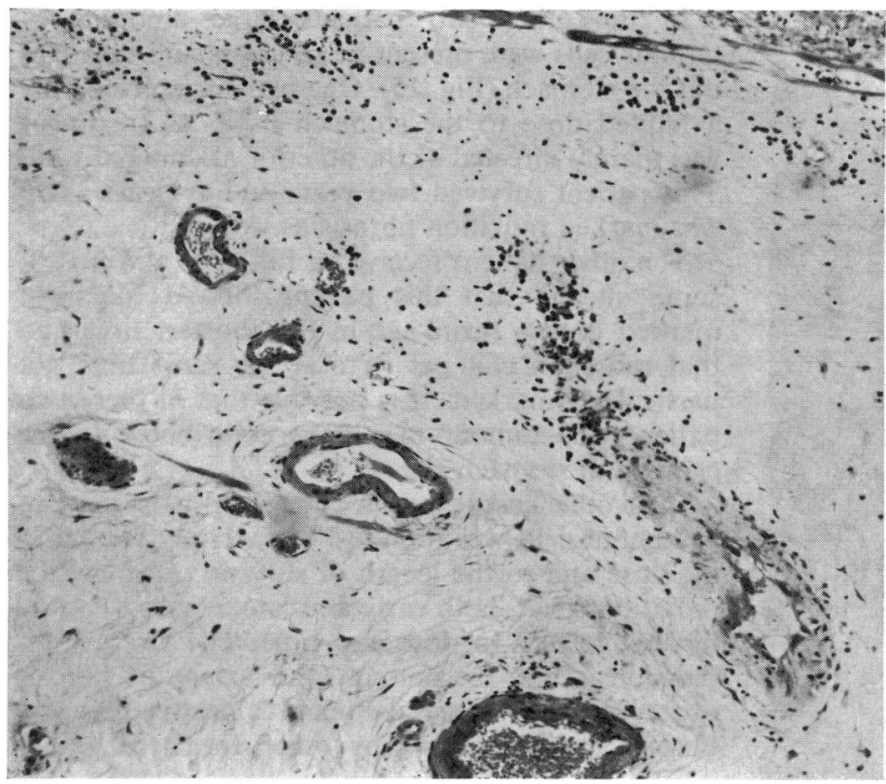

Fig. 9 Submucosa with early radiation effect including oedema and dilatation of blood vessels, some of which show fibrin thrombi in the lumina. $H$ and $E, \times 90$.

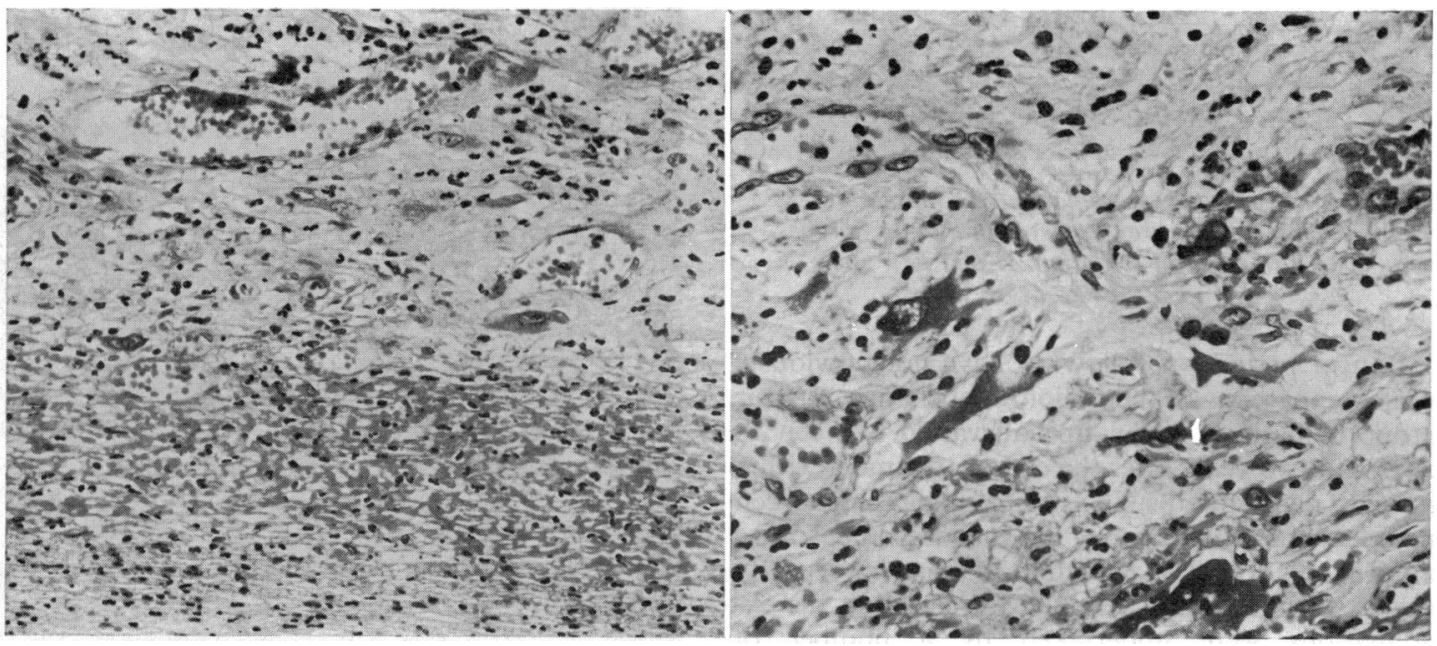

FIG. 10

FIG. 11

Fig. 10 A low power photomicrograph of the radiation fibroblasts admixed with inflammatory cells in an oedematous submucosa. $H$ and $E, \times 275$.

Fig. 11 A high power photomicrograph of bizarre radiation fibroblasts. $H$ and $E, \times 562$.

remarkable difference in the magnitude of damage to the duodenal mucosa occurring within the first 72 hours.

Early information on microscopical data came from patients dying in the first few days after the Hiroshima and Nagasaki atomic bomb explosions (Oughterson and Warren, 1956). Ulcerative lesions with focal necrosis and masses of numerous bacteria were noted after seven to 11 days in patients exposed within $1371 \mathrm{~m}(1500 \mathrm{yd})$ of the hypocentre and suffering from severe burns.

Intestinal mucosa has been shown to recover after a dose of $2000 r$ of $x$-rays, the appearances returning to normal within 14 days of ending treatment. 


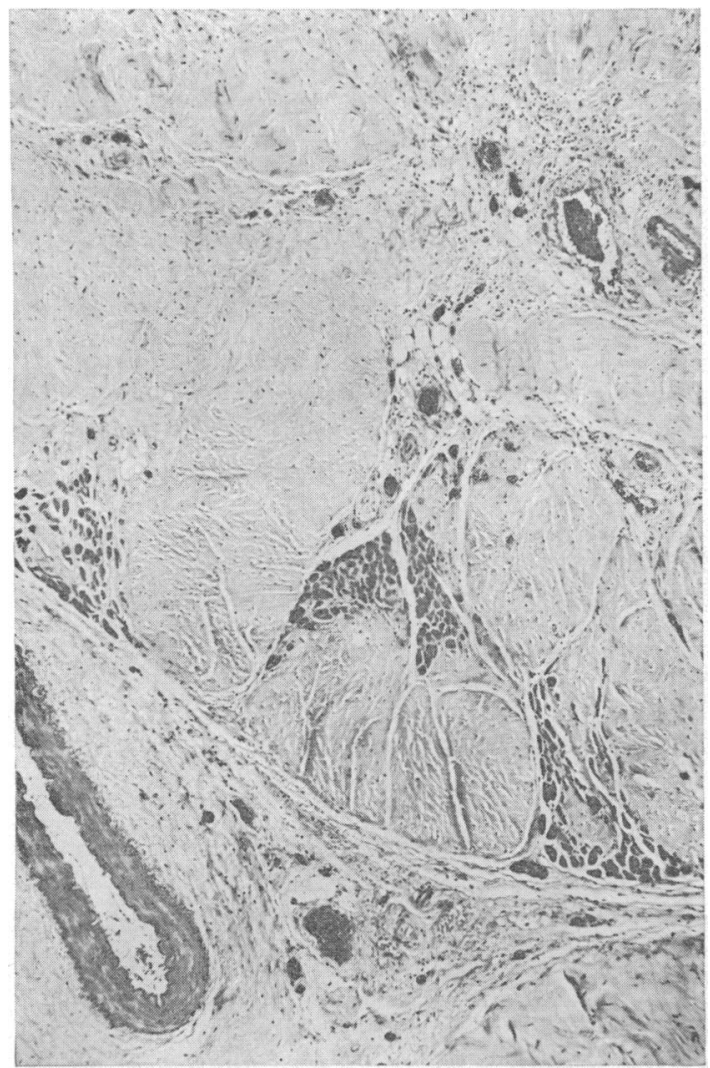

Fig. 12 Widespread replacement of the muscle wall of the stomach (the dark grey areas) with mature fibrous tissue as a late effect of radiation. $H$ and $E, \times 68$.

Longer periods of follow-up have not previously been compared with the radiological changes. In our group of patients follow-up had varied between 10 days and two years after the end of treatment with a mean of eight months. Consistent changes have been noted in the stomach and the mucosal appearance after irradiation. The reduction in stomach capacity was a constant finding despite the fact that the examinations were undertaken by a number of different radiologists. By choosing similar projections and correcting for the magnification effect we have eliminated a number of potential sources of error.

Of the 10 patients included in this group only five were examined histologically. Three of the five received a high dose and all of them showed considerable amounts of mature fibrous tissue. The number of tumour cells was small and was not seen in all blocks. It was predominantly present in the submucosa and subserosa (Fig. 14). In these cases, therefore, the changes in stomach size could not have been affected by such a small number of tumour cells.
In the other two patients large amounts of tumour cells were present in all layers and throughout the stomach (Fig. 15). One patient received only a limited dose to the stomach itself, as treatment was mainly directed at the anterior abdominal wall. This patient survived two years and at death there was marked radiation fibrosis as well.

The other patient received a full dose and it is of some interest that this patient showed the most marked gastric shrinkage in the shortest time and that radiation changes were at the same time not particularly marked. It is possible that in these two patients the tumour may have contributed to the changes in stomach size.

In all other cases examined at necropsy the amount of tumour cells was found to be inversely related to the dose and to the length of survival, whereas the radiation effect, both early and late, appeared to be directly related to dose and time. The overall impression is that the amount and extent of fibrosis observed in the material studied is greater than the fibrosis usually caused by other forms of radio-

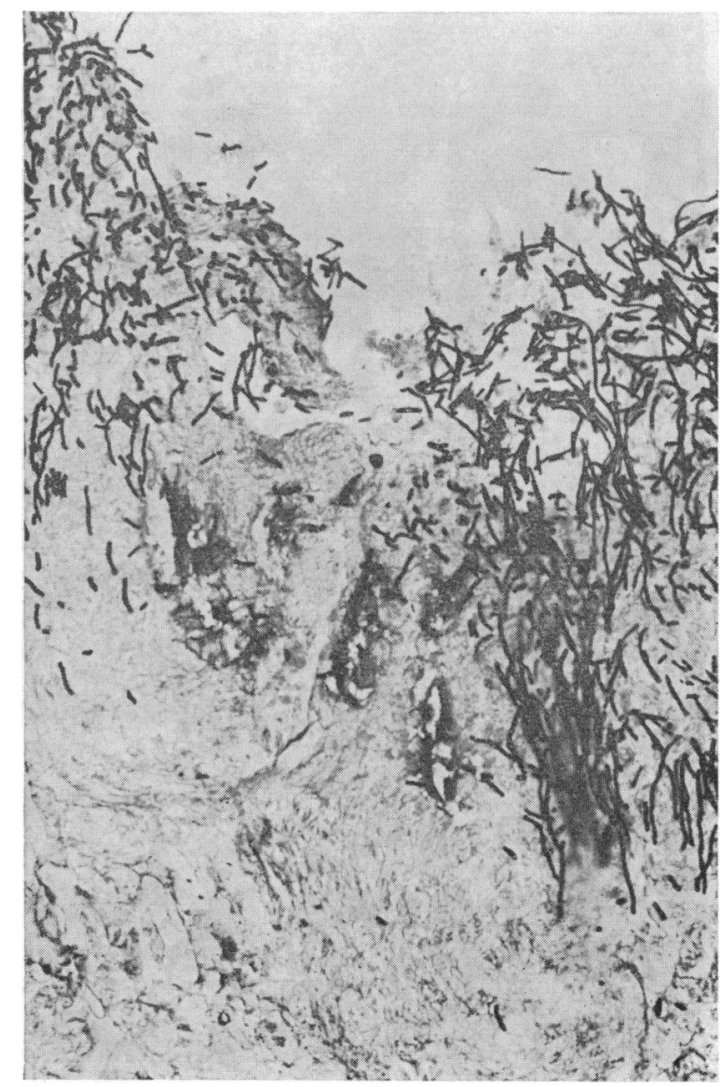

Fig. 13 Periodic acid-Schiff (PAS) positive heavy fungal infection of ulcerated mucosa. AB/PAS, $\times 250$. 


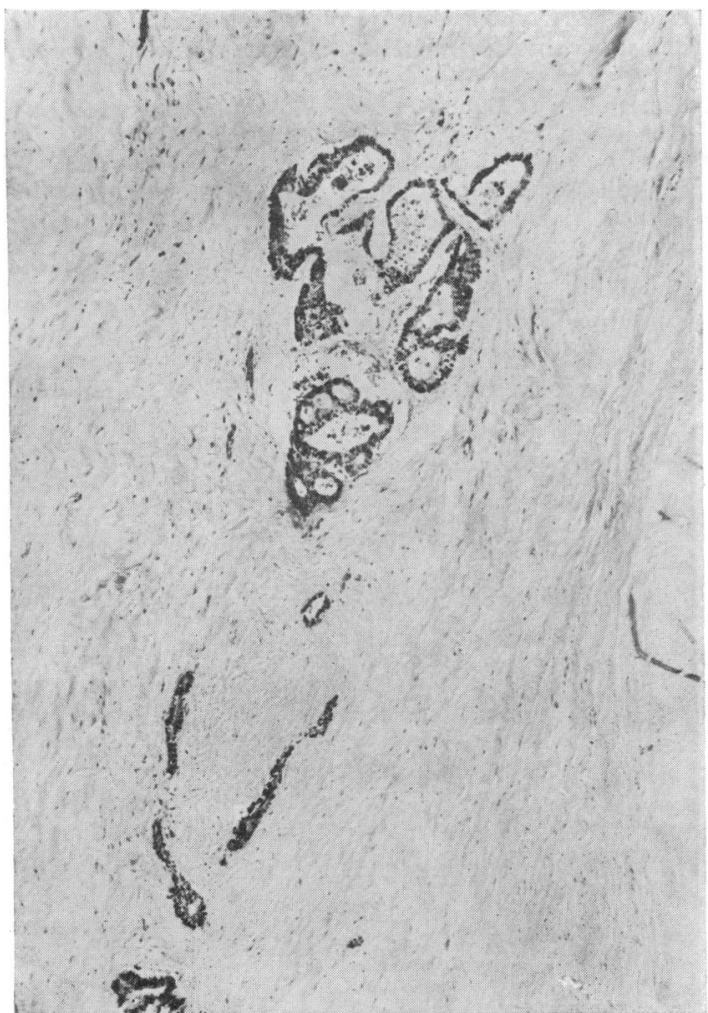

Fig. 14 Residual clusters of well-differentiated adenocarcinoma in the submucosa of the stomach. $H$ and $E, \times 56$.

therapy and it is likely that, in the majority of cases, neutron radiation rather than tumour was the main factor contributing to the shrinkage of the stomach. Nevertheless, we feel that such an observation must be investigated by detailed comparative studies.

The loss of normal rugal pattern with development of shallow, irregular, and widely spaced clefts was seen to persist in those areas of the stomach which received less than the full dose. Its appearance 10 weeks after the end of irradiation may have coincided with irregular and unequal rates of regeneration of the intestinal mucosa in various areas affected to different degrees of severity. When such changes were present at the time of death the histological appearances were unremarkable, there being little necrosis or ulceration.

The more marked changes occurred later and were always associated with a dose of about $1560 \mathrm{r}$. In such patients marked ulceration was present microscopically. In view of the absence of any histological evidence of mucosal hyperplasia, these radiological changes were probably due to ulceration and

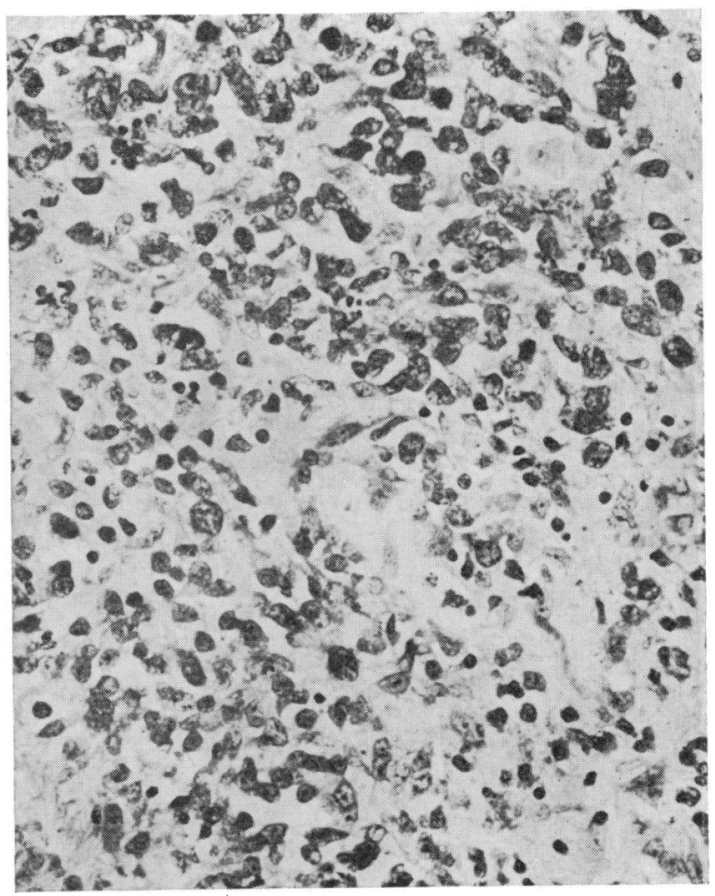

Fig. 15 Extensive infiltration of viable tumour cells many of which show mitotic activity. $H$ and $E, \times 275$.

necrosis interspersed with areas of relatively normal mucosa, the whole surface layer being altered to some extent by underlying submucosal fibrosis. There is no evidence that tumour contributed directly to the mucosal appearances except possibly immediately after treatment.

That the macroscopic tumour can be eliminated has been demonstrated; however, microscopical clumps of tumour cells remain in the deeper layers of the stomach wall and these may give rise to recurrence if the patients lived long enough.

In the present series, cases were advanced before the treatment was begun and in most patients distant metastases were the cause of death fairly soon after treatment.

\section{References}

Bewley, D. K. (1976). British Journal of Radiology (In press). Burn, J. I. (1971). Cancer of the stomach and oesophagus. British Journal of Surgery, 58, 798-700.

Catterall, M., Kingsley, D., Lawrence, G., Grainger, J., and Spencer, J. (1975). The effects of fast neutrons on inoperable carcinoma of the stomach. Gut, 16, 150-156.

Gilbertsen, V. A. (1969). Results of treatment of stomach cancer. Cancer, 23, 1305-1308.

Hirose, F. (1967). Acute radiation damage to the digestive tract: a comparison of effects of fast neutrons and $x$-rays. Acta Pathologica Japonica, 17, 324-327. 
Inberg, M. V., Vuori, J., and Viikari, S. J. (1972). Carcinoma of the stomach: a follow-up study of 1963 patients. Acta Chirurgica Scandinavica, 138, 195-201.

Lumpkin, W. M., Crow, R. L., Jr, Hernandez, C. M., and Cohn, I. Jr (1964). Carcinoma of the stomach: a review of 1,035 cases. Annals of Surgery, 159, 919-932.

Oughterson, A. W., and Warren, S. (1956). Medical Effects of the Atomic Bomb in Japan. McGraw Hill: New York.
ReMine,W. H., and Priestley, J. T. (1966). Trends in prognosis and surgical treatment of cancer of the stomach. Annals of Surgery, 163, 736-745.

Takahashi, S. (1969). An Atlas of Axial Transverse Tomography and its Clinical Application. Springer, Berlin.

Zacho, A., and Fischermann, K. (1966). The results of surgical treatment of cancer of the stomach. Surgery, Gynecology, and Obstetrics, 123, 73-79. 